\title{
Novel Agent Based-approach for Industrial Diagnosis: A Combined Use between Case-based Reasoning and Similarity Measures
}

\author{
Fatima Zohra Benkaddour ${ }^{1}$, Noria Taghezout ${ }^{1}$, and Bouabdellah Ascar ${ }^{2}$ \\ 'Laboratoire d'Informatique Oran (LIO), Département d'Informatique, University of Oran1 Ahmed BenBella, BP \\ 1524 EL Mnaouer Oran, Algeria \\ ${ }^{2}$ INOTIS enterprise, Oran, Algeria
}

\begin{abstract}
In spunlace nonwovens industry, the maintenance task is very complex, it requires experts and operators collaboration. In this paper, we propose a new approach integrating an agentbased modelling with case-based reasoning that utilizes similarity measures and preferences module. The main purpose of our study is to compare and evaluate the most suitable similarity measure for our case. Furthermore, operators that are usually geographically dispersed, have to collaborate and negotiate to achieve mutual agreements, especially when their proposals (diagnosis) lead to a conflicting situation. The experimentation shows that the suggested agent-based approach is very interesting and efficient for operators and experts who collaborate in INOTIS enterprise.
\end{abstract}

Keywords -Agent, Case-based Reasoning, Collaboration, Diagnosis, INOTIS, Similarity Measures.

\section{INTRODUCTION}

$\mathrm{I}_{\mathrm{n}}^{\mathrm{N}}$ $\mathrm{N}$ the industrial processes, the major failures that are related to Imachines' breakdowns have an impact on the production process, especially, when a corresponding diagnosis has been established on the right time. The anticipation of the failure at the beginning of its appearance can avoid dysfunctions and breakdowns.

The increasing complexity of automated industrial systems and competitiveness constraints in terms of production cost, availability, and safety of installations, have mobilized in recent years a large community of researchers to improve the monitoring and diagnosis of such processes. The diagnosis is a research theme addressed by several scientific communities (Automatic Computing, Industrial Engineering ...). It is nowadays the heart of industrial concerns [1].

Our research is oriented towards the nonwovens industry and «INOTIS» enterprise which is the candidate for our study. This enterprise was established in 2003, its major mission is to develop manufacture and sell world-class spunlace fabric for critical environments, where contaminated control and comfort of the use are of vital importance. This will be achieved through cost-effective production, consistency, and reliability. Besides, it provides a high level of both service and quality [2].

In fact, the market of nonwovens bonded by water jet (also called spunlace or hydroentanglement nonwovens) showed in the last years a considerable growth rate, which will also grow strongly in the future. These products are mainly used in the medical and hygienic field, but also the technical applications are being more and more important.

In spite of a very versatile range of applications, opportunities for growth exist in the market for hydroentangled (spunlaced) nonwovens, but they can only be continuously realized by the innovative introduction of products following the market's needs.

In [3], authors designed the first Collaborative Decision Support System «CDSS» to deal with complex decision problems that are related to spunlace nonwovens industry.

This system was based on a collaborative decision making process that exploits the represented knowledge in domain ontology and uses a case-based reasoning. CDSS proposed a collaborative platform that relies on Web 2.0 technologies and allowed operators, production managers, or experts to share and exchange experiences in industrial diagnosis such as machines 'breakdowns.

\section{A. Major contribution}

The aim of this study is to provide a multi agents system for solving industrial diagnostic problems. It encourages industrial nonwovens operators to collaborate and find solutions to their problems without resorting to the domain expert in a quick and efficient manner.

Our contribution deals mainly with two aspects:

1. We propose, an agent-based approach to provide a classification of the solutions given by the nonwoven operators in order to solve a new problem. Nonwovens operators don't only want to get solutions to their problems, but they also required the relevant solutions in the top positions of the list. We set up a method for ranking the solutions proposed by these operators using nonwovens domain ontology. This method is based on the information content of solutions and operators' preferences (vote), in which this later provides more relevant rankings.

2. We conduct a comparative study to choose a suitable similarity measure and improve the case-based reasoning results in order to measure the degree of similarity (or dissimilarity) between the target case and cases sources; our cases-base contains some cases of industrial diagnosis that have been registered since 2009 to INOTIS enterprise. These cases were recovered from meetings, and interviews after being filtered and reformulated.

\section{B. Paper organization}

This paper is organized as the following: In Section 2, we describe some related works. In Section 3, we present our approach: Firstly, we explain how we use case-based reasoning. We give a detailed description of the multi agents system. Then, we present the negotiation protocol. In section 4, we present a discussion of the obtained results. Finally, we conclude with some future works which are outlined in section 5. 


\section{BACKGROUND AND Related Work}

In this section, we analyze some works which are related to several approaches that integrate case-based reasoning and multi-agent system for industrial diagnosis. Through this study, we address the following summary.

\section{A. Case-based reasoning systems}

The case-based reasoning is the nearest of human reasoning. It provides the ability to make decisions in the way human beings take in the real time. The case-based reasoning allows reasoning on issues already resolved and stored in the cases-base. Past experiences are reused to solve new problems. The learning process is easier to be treated in the approach of case-based reasoning [4].

Another major advantage of this approach over other approaches of reasoning, is the value of the solution. In the adaptation phase, the proposed solution is revised and adapted to the problem constraints; this phase enhances the solutions and makes an effective case-based reasoning. The errors of the previous solutions do not propagate into future ones [5].

In [6], authors developed a cost model for the conceptual phases of Case Based Reasoning (CBR). This model used the concept of Euclidean distance for the similarity measure and Genetic Algorithms for the optimization of the weights of attributes.

In [7], authors used a cases-base for diagnosis and industrial maintenance using an ontology. Each CBR' phase is implemented by including the adaptation phase that uses decision rules. The authors used an ontology to characterize a case (problem) and the comparison between the cases was done by applying a weighted measure of similarity.

In [8], authors proposed an approach based on case-based reasoning (3R model) with a three phases cycle (Researching, Retrieving, and Retaining). They calculated the optimum weight of the attributes of each case automatically in order to extract the similar cases and deliver the final solution. They proposed a model for negotiation strategy to predict the behavior of the seller.

In order to evaluate the equipment maintenance support effectiveness, a conducted research was made by [9]. Their focus was basically structured on case-based reasoning. During this process, they used the representation and storage methods, where they analyzed the equipment maintenance case.

The work presented in [10], was focusing on the decision-making processes that are involved in maintenance. Authors proposed a knowledge operating approach, which is used in case-based reasoning system for the resolution of maintenance problems. This approach depended on the extension of the similarity measure on the basis of some case descriptors.

\section{B. Multi-agent systems for industrial diagnosis}

It is impossible to content with a centralized and rigid approach to design a diagnostic system. Distribute the diagnostic procedure seems obvious in this case. Consequently, the idea appears in order to multiply intelligent entities and to equip them with communication skills. These entities can communicate with each other and cooperate to build a solution for diagnosis problem. Multi-agent system is adapted to this problematic. In fact, the agent contributes to solve a problem. It communicates with several agents in order to emerge towards a comprehensive solution. Multi-agent approach makes the solution implementation possible same as a human organization. The first advantage to use this paradigm is to lead modular systems where adding an agent or modifying the structure of a system does not induce a Re-design of a solution, but converges automatically to a new comprehensive solution. The second advantage is to decompose complex problems to some elementary problems and create capable entities to solve them by cooperating [1].

Three phase induction motors were presented by [11] in order to improve and identify faults by means of a multi-agents system.

In the future, distribution and complexity of processes must be handled by the fault diagnosis systems. Authors in [12] described the fault detection and isolation (FDI) agents, where the reactive layer was fundamentally structured on the decomposition wavelet methods.

Much attention has been given to designing the process in [13] a framework. They masterfully presented an essential element of mechanism design for a multi-agent system.

In [1] authors provided an multi-agent system for the collective diagnosis of complex systems using the technique of logical diagnosis based on consistency with a particular interest in the distribution of diagnostic analysis stage.

Multi-agent systems can be used in several domains. We cite below someone.

The study presented in [14] deals with an automated multi-agent negotiation framework in order to elaborate decision making in the construction domain. The proposed framework was composed of negotiation algorithm, negotiation style, negotiation protocol, and solution generators that were integrated into agent architecture. Furthermore, negotiation base and the conflict resolution algorithm, were blended within the environment in order to facilitate the negotiation process. The proposed architecture enabled software agents to conduct negotiations and autonomously make decisions.

Authors presented in their work a model that is based on multi-agent system active between the user and grid client as Globus Resource Allocation Manager (GRAM). They displayed a designing scheduler process by using multi-agent systems. To achieve their goal, they used the hidden Markov models for the matchmaker process and Telecom Italia Lab approach for developing their system [15].

In [16], authors was interested in the integration of web service. They presented storage solutions which were based on a dynamic partial replication and they proposed a distributed architecture by using some agents in order to stand for this mechanism.

\section{Methodology}

In this section, we present the main aspect of our contribution.

\section{A. Case-based reasoning}

To solve the problems of the nonwovens industry, we utilize previous situations which were stored in the cases-base. To cope with new problems, we already used solved past problems that have a large degree of similarity comparing to current situations (new problems). We give more details in the following subsections as shown in Fig. 1.

\section{1) Cases-base}

To perform any inference task, reasoning engine requires knowledge of the target area. In the case-based reasoning system, this knowledge is taken from the cases-base.

Our CBR system starts reasoning from a number of cases. These cases cover the target area very well consequently, interesting solutions are returned. We have recovered industrial diagnosis cases from $\mathrm{Pv}(\mathrm{s})$ and annual interviews of INOTIS company.

\section{2) Indexing case}

Case-based reasoning system is interesting only if it has a significant cases-base, we obviously considered indexing event cases as a solution 
to find similar cases quickly.

There are many ways to order cases, we opted for indexing by keywords which are using a textual representation for the case description.

Cases are indexed and ranked in the cases-base according to the keywords that are given in their description. Thus, the keywords contained in the event table are used to locate the corresponding event.

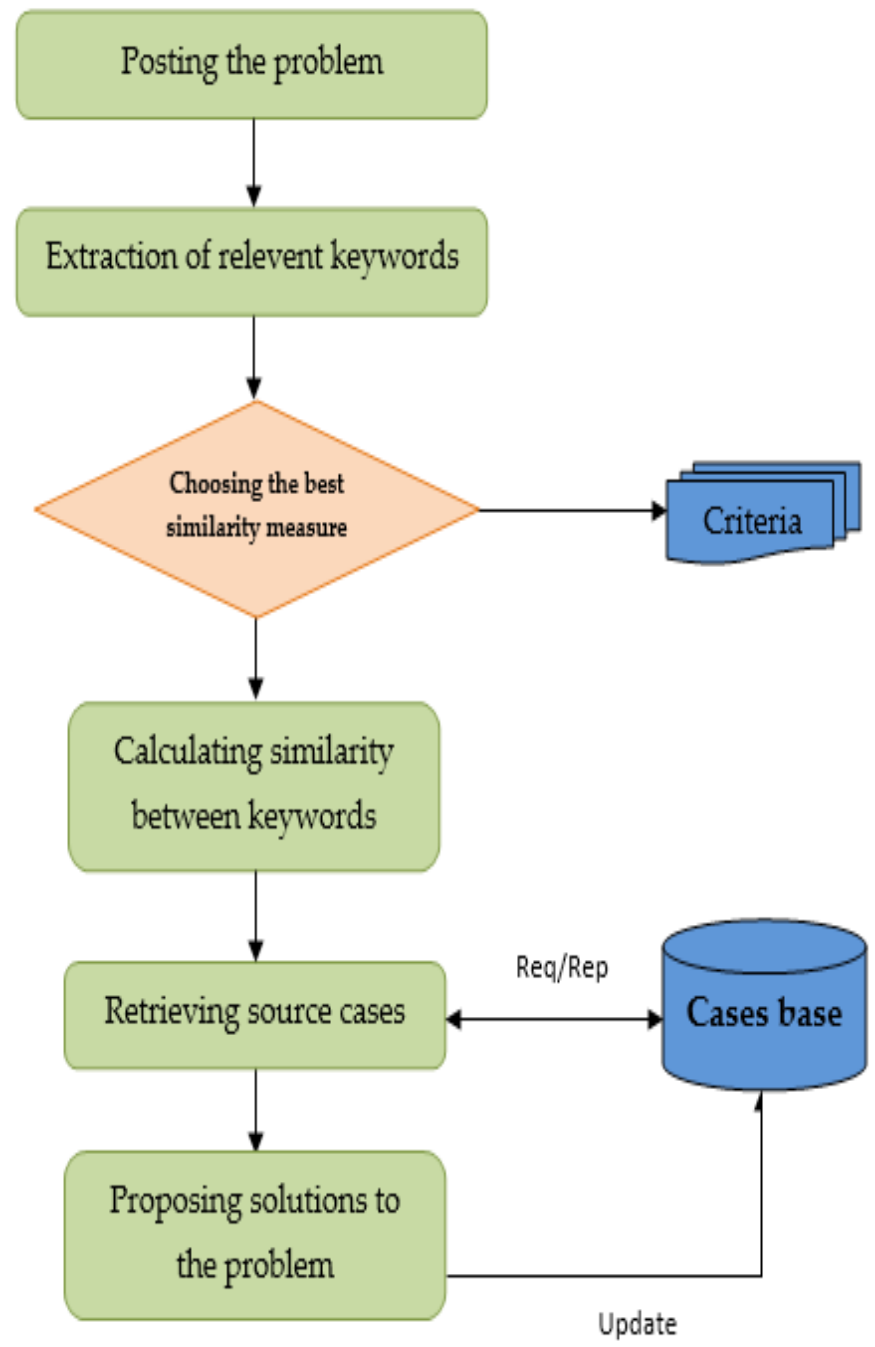

Fig. 1. Researching solutions using CBR system.

\section{3) Case-based reasoning process}

The case-based reasoning aims to solve new problems by comparing them to source cases that are recorded in the cases-base. In our work, we use 3R model represented in three (3) main phases (Representing, Reusing, and Retaining). These phases can be described as the following.

1. Representing a new problem (target case): That allows acquisition of any relevant information that describes the new problem (relevant words). This phase gives the system an initial description. It includes: structuring, modeling, and case representation. This latter is presented in a similar manner to a source case.

2. Reusing cases (sources): Corresponds to search for the most similar cases. This phase maps the descriptors source cases with descriptors of the target case. We use the technique of calculating the degree of matching descriptors (similarity between both cases) to achieve this objective. We justify a used similarity measure in the comparative study.

3. Retaining case: In this step, the target case is added to the cases-base which cases-base is synthesized, and amended where arguably the system can learn new skills.

\section{B. Multi agent system}

We propose a multi-agent system which is composed of four (4) agents, in which each one has a specific role as shown in Fig. 2.

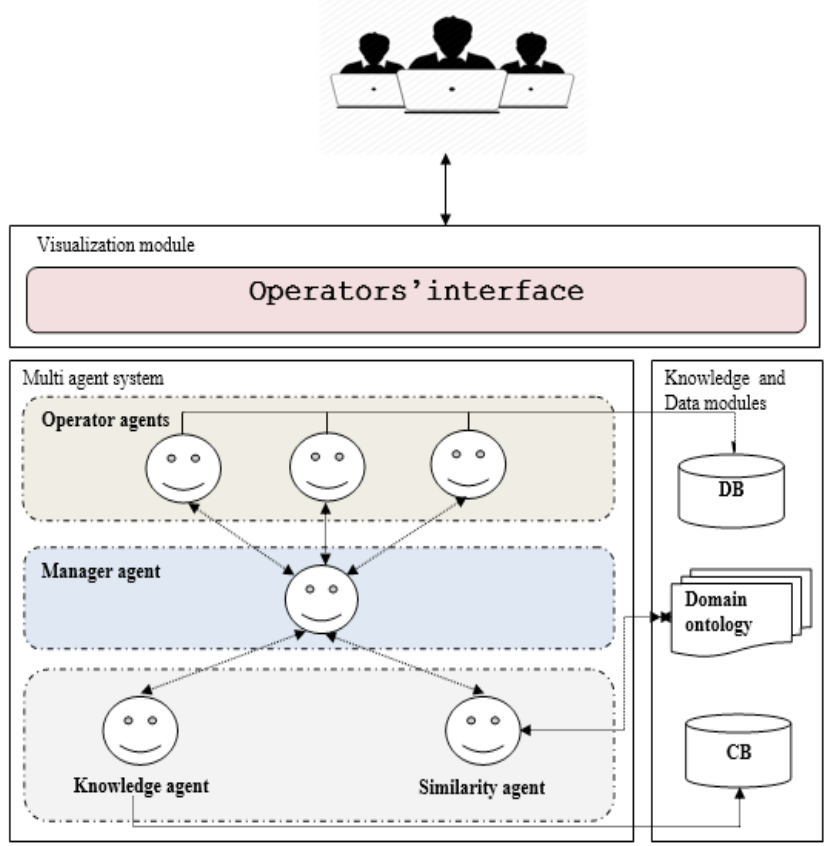

Fig. 2. Our multi agent system.

\section{1) Agents}

Below, we describe each agent and its aim.

1. Operator agent: This agent is created when operator connects to the platform. It analyzes and decomposes the operator's message (Problem and / or solution) into a set of relevant words (see Fig. 3). It scores the solutions according to its preferences introduced through the preferences module. Operator agent retrieves and recommends some solutions to the operators.

Algorithm 1: Extracting relevant elements ( $\downarrow \mathrm{Pbm}, \downarrow$ Keywords, $\uparrow$ List_MP)

String Txt; List Words;

Begin

Txt $\leftarrow$ Elimination of stop words ( $\mathrm{Pbm})$

Words $\leftarrow$ Searching_synonymes (Words);

Words $\leftarrow$ Elimination words of very high occurrence (Words);

List_MP $\leftarrow$ Words + Keywords;

End

Fig. 3. Extracting relevant elements algorithm.

2. Manager agent: In addition to managing agents, it prompts similarity agent to pre-order the list of proposals (solutions). Then, it invites operator agents to vote. The operator should score solutions before the deadline and the best mark of solution is $10 / 10$ (criteria). After that, it diffuses subsequently an ordered list of solutions. The vote is detailed in Fig. 4. 


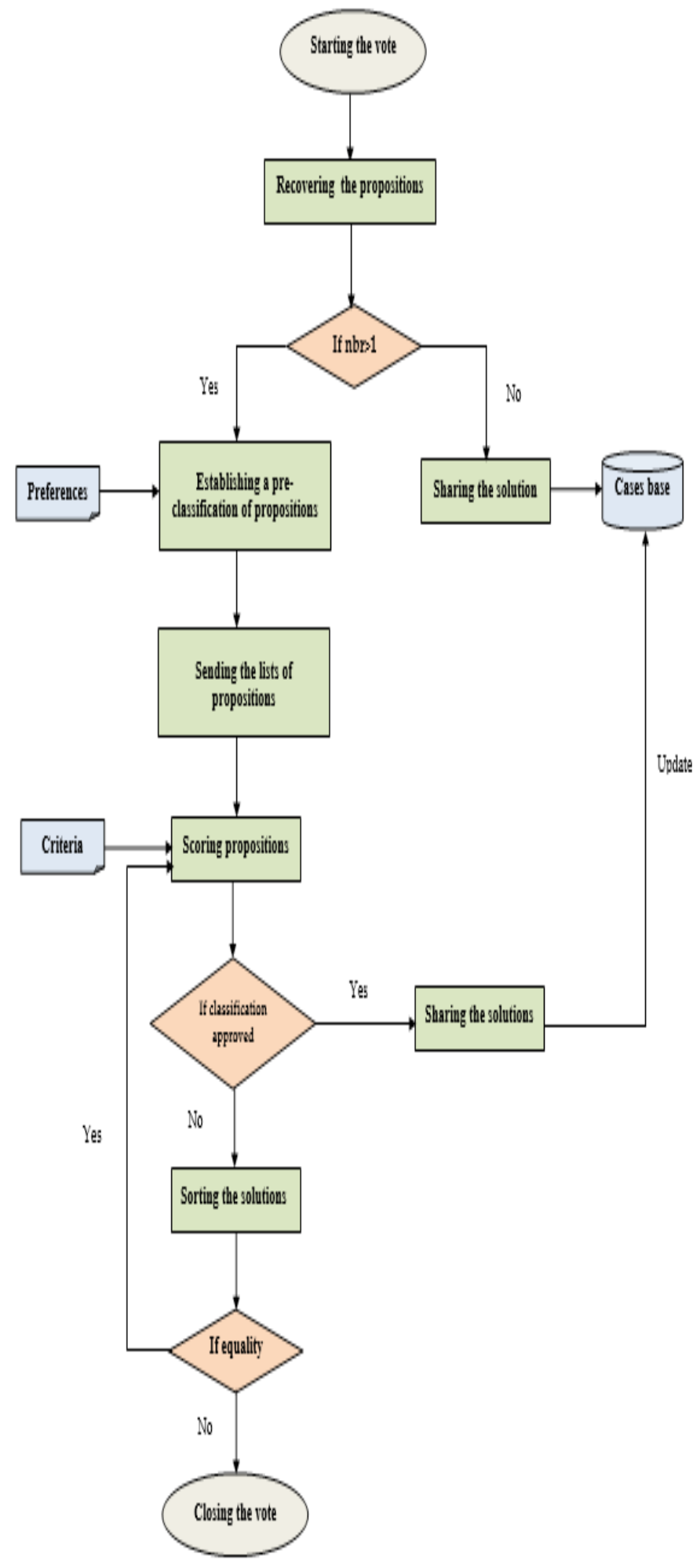

Fig. 4. Steps of the vote.

3. Similarity agent: it has two main functions: First, it compares between target case and source cases using a similarity measure. Second, it orders the list of proposals sent by the manager agent. This order is carried out according to the information content of the proposals.

4. Knowledge agent: It retrieves the similarity results calculated by the similarity agent and recovers solutions from CB (see Fig. 5), then it updates the cases-base.

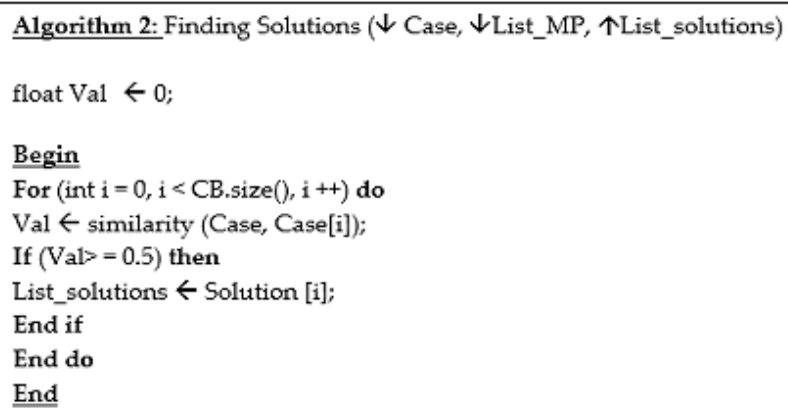

Fig. 5. Finding solutions algorithm.

\section{2) Interaction between agents}

The agents act by groups, and they are characterized by the sharing tasks. This collaboration requires their interaction.

An agent (operator, similarity or knowledge) sends a message in two cases: When receiving a message from another agent (data reception required to process), during the execution of an action.

In addition, the manager agent sends messages when it starts voting.

Table 1 shows the types of messages exchanged between agents.

TABLE I

Exchanged Messages Between Agents

\begin{tabular}{|c|c|c|c|}
\hline Action & Sender & Receiver & Content \\
\hline Inform & Operator agent & Manager agent & Life message \\
\hline Propuest & Manager agent & $\begin{array}{c}\text { Similarity } \\
\text { agent, } \\
\text { Knowledge, } \\
\text { operator agent }\end{array}$ & $\begin{array}{c}\text { Preorder list } \\
\text { of solution, } \\
\text { update CB, need } \\
\text { solution }\end{array}$ \\
\hline Confirm & Manager agent & Operator agent & Scoring solutions \\
\hline
\end{tabular}

\section{Negotiation protocol}

The proposed negotiation protocol is characterized by a succession of messages exchanged among the agents; it is divided into three important phases:

1. Distribution of the problem: it is the first phase of our negotiation protocol, it initiates the negotiation. The message is sent by the manager to all operator agents that are considered to be able to carry out the task. Operator agents propose solutions and after that, they send them to the manager.

2. The conversation phase: it is the second phase, where the manager agent requests similarity agent to establish a preordered list of propositions. This pre-order is realized with similarity measure. Similarity agent calculates information content of each proposition based on domain ontology. Then, the manager agent shares the list of solutions with the operator agents. According to operator agents' preferences, they establish a score and attribute it to each proposition and return the list to the manager agent.

3. Final decision: It is the third and the final phase where the manager agent gathers all its received scores and organizes the final list of solutions. If two solutions are equals, it invites operator agents to vote with a restricted list of solutions.

The agent's strategies are defined in the sequence diagram shown in Fig. 6. 


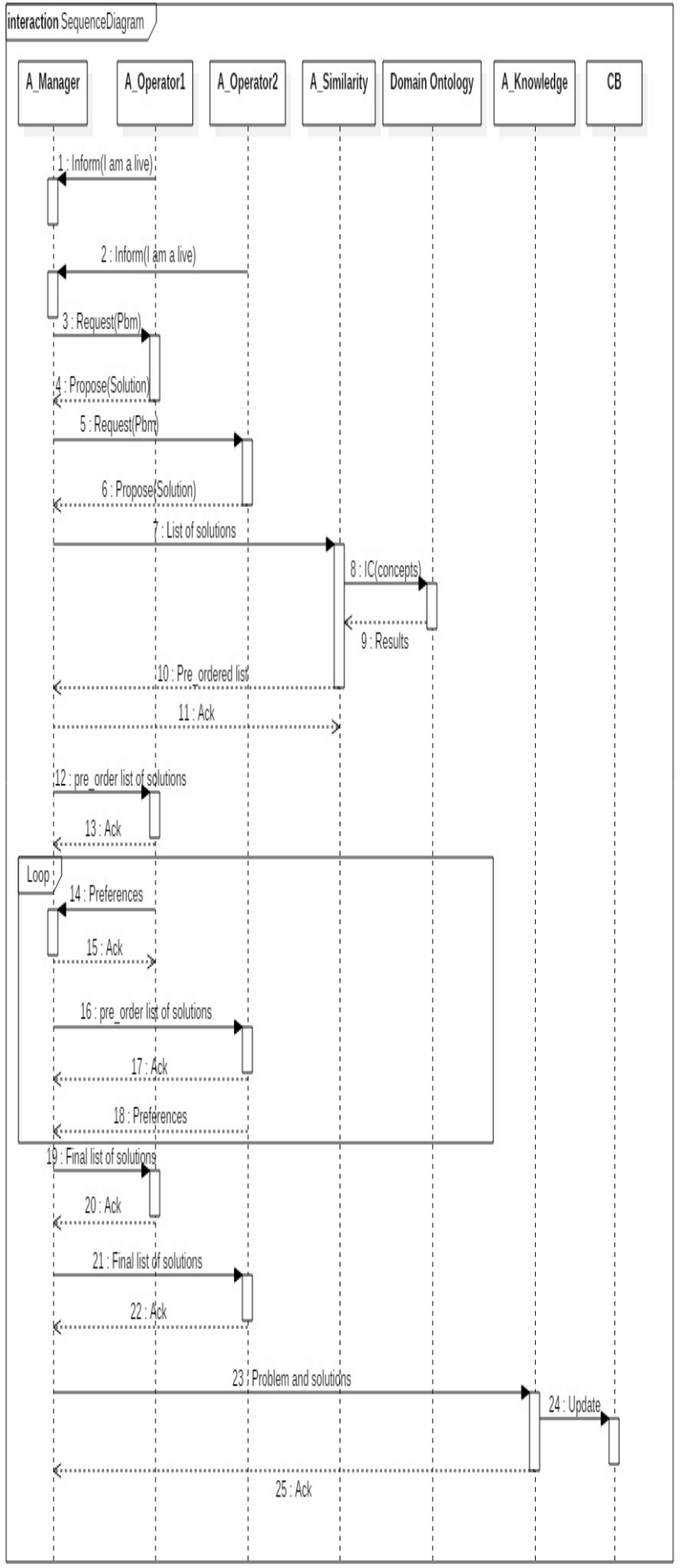

Fig. 6. Time sequence of the multi-agent process with AUML.

\section{1) Negotiation cardinality}

Our protocol is based on the exchange of messages between a manager and several operators, so it allows negotiations from 1 to $\mathrm{n}$. We allow the simultaneous filing solutions of several problems. Otherwise, many manager agents can simultaneously propose a vote to operator agents, it is therefore negotiating $\mathrm{n}$ to $\mathrm{m}$ agents, more accurately $\mathrm{n}$ simultaneous negotiations 1 to $\mathrm{m}$ agents.

\section{2) Default response time}

During negotiations, operator might not respond to the manager proposal because he is absent, or a fault has occurred.

It is necessary to continue, for that reason an answer waiting time mechanism is in place, and when that time is up, the manager considers a default response for the operator. This default response will be the average scores provided by the rest of operators. In fact, waiting time is limited to 60 minutes.

\section{3) Number of responses needed for ranking solutions}

In order to classify the solutions, the manager need all operators 'responses. When the operators don't reply, the manager takes the average score, for each solution as a default response.

\section{4) Scenario 1 (without conflict)}

Here, we present a concrete example to illustrate the operation of the vote. The operators 'notes are between 1 and 10 which is considered as the best possible rating for a solution. The solutions ranking is therefore obtained from taking solutions in descending order from the highest rating to the lowest.

In this example, four solutions are presented: $\mathrm{S} 1$ to $\mathrm{S} 4$, the manager agent invites three operators to vote, namely $\mathrm{O} 1, \mathrm{O} 2$ and $\mathrm{O} 3$. It sends the solutions list to similarity agent. This latter establishes a ranking of solutions by giving a score to each one.

The following table shows respective solutions 'notes for each operator and the order established by the similarity agent.

TABLE II

Solution's Score Given by Several Agents

\begin{tabular}{|c|c|c|c|c|}
\hline Solution & Similarity agent & O1 & O2 & O3 \\
\hline S1 & 10 & 8 & 1 & 10 \\
\hline S2 & 8 & 10 & 7 & 8 \\
\hline S3 & 5 & 9 & 10 & 2 \\
\hline S4 & 2 & 3 & 9 & 5 \\
\hline
\end{tabular}

From Table 2, the order that is given by the similarity agent is $\{\mathrm{S} 1$, $\mathrm{S} 2, \mathrm{~S} 3, \mathrm{~S} 4\}$, this order is based on similarity measure. First, operator 1 classifies the solutions in this way $\{\mathrm{S} 2, \mathrm{~S} 3, \mathrm{~S} 1, \mathrm{~S} 4\}$. Second, operator 2 prefers this one $\{\mathrm{S} 3, \mathrm{~S} 4, \mathrm{~S} 2, \mathrm{~S} 1\}$. Finally, operator 3 gives the following order: $\{\mathrm{S} 1, \mathrm{~S} 2, \mathrm{~S} 4, \mathrm{~S} 3\}$.

After the vote, the manager agent calculates the score of each solution according to the following formula:

$$
\operatorname{Score}\left(S_{i}\right)=\sum_{j=0}^{n} \text { note }_{j}
$$

Where $\mathbf{n}$ is the number of participants in the vote (operators and the similarity agent) the similarity agent intervenes only during the first round of the vote.

$\mathbf{i}$ is the solution on which participants must vote.

note $_{\mathbf{j}}$ Is the score given by each participant.

Score $(\mathrm{S} 1)=10+8+1+10=29$

Score $(\mathrm{S} 2)=8+10+7+8=33$

Score $(\mathrm{S} 3)=5+9+10+2=26$

Score $(\mathrm{S} 4)=2+3+9+5=19$

After voting, the manager agent notifies the other agents with the following list of solutions: $\{\mathrm{S} 2, \mathrm{~S} 1, \mathrm{~S} 3, \mathrm{~S} 4\}$.

To be more precise, the significance of the obtained solutions is 
given in Table 3.

TABLE III

Solutions Collected from Operators

\begin{tabular}{|c|c|}
\hline Number & Solution \\
\hline S1 & Change the bladder \\
\hline$S 2$ & Check the communication and power part \\
\hline$S 3$ & Drain the strainer \\
\hline S4 & Measure the PH of process water \\
\hline
\end{tabular}

\section{5) Scenario 2 (with conflict)}

Let us consider now the case of equal solutions. Here, the manager agent starts for the second time the vote only between operators. The results are described in Table 4.

TABLE IV

Priority of Solution Given by SeVeral Agents

\begin{tabular}{|c|c|c|c|c|}
\hline Solution & Similarity agent & O1 & O2 & O3 \\
\hline S1 & 10 & 8 & 1 & 10 \\
\hline S2 & 8 & 10 & 7 & 8 \\
\hline S3 & 6 & 9 & 10 & 4 \\
\hline S4 & 2 & 3 & 9 & 5 \\
\hline
\end{tabular}

Score $(\mathrm{S} 1)=10+8+1+10=29$

Score $(\mathrm{S} 2)=8+10+7+8=33$

Score $(\mathrm{S} 3)=5+9+10+2=29$

Score $(\mathrm{S} 4)=2+3+9+5=19$

$\mathrm{S} 1$ and S3 are equal since both of them have the same score $(=29)$. The manager agent restarts again the vote between operators for S1 and S3. The corresponding results are shown in Table 5.

TABLE V

Priority of Solution Given by Several Agents

\begin{tabular}{|c|c|c|c|}
\hline Solution & O1 & O2 & O3 \\
\hline S1 & 9 & 10 & 7 \\
\hline S3 & 8 & 6 & 10 \\
\hline
\end{tabular}

Score $(\mathrm{S} 1)=9+10+7=26$

Score $(\mathrm{S} 3)=8+6+10=24$

The final ranking of solutions becomes: $\{\mathrm{S} 2, \mathrm{~S} 1, \mathrm{~S} 3$, and S4\}.

1. Checking the communication and power part,

2. Changing the bladder,

3. Draining the strainer,

4. Measuring the $\mathrm{PH}$ of process water.

This scoring formula takes into account the preferences of each operator and the similarity agent proposal for each suggested solution.

\section{RESULTS AND DISCUSSION}

\section{A. Comparison between semantic similarity measures}

Below, we propose a comparative study between three (3) semantic similarity measures [17], [18], and [19]. To retrieve similar cases in a cases-base, we focus on some keywords that are specific to nonwovens domain.

Thus, the dispersion of the analysis is reduced. This approach is primarily depending on the ontology that we have developed. To determine the best similarity measure, we conducted a comparative study between the semantic similarity measures specifically semantic measures based on taxonomic distance.
We took a sample of twenty (20) cases most representative sources cases from CB (Cases-Base) including eight (8) relevant cases and eleven (11) irrelevant. Knowing that a relevant case is a matter of couple and a single solution provided from experts; an irrelevant case is a couple of problem and some solutions, Fig.7 shows that sample. To better explain this comparative study, we took an example for each measure. According to several experiments, we concluded that the suitable similarity threshold in this study is 0.5 , a threshold higher than 0.5 will give the same solutions for each problem (shown in Table 6).

TABLE VI

Cases Chosen to Illustrate the Experience

\begin{tabular}{|c|c|c|c|}
\hline- & Id & Problem & Keywords \\
\hline Source case & 6 & $\begin{array}{c}\text { Nitrogen gas } \\
\text { leak }\end{array}$ & $\begin{array}{c}\text { Pompe_HP, } \\
\text { HP2, Vessie }\end{array}$ \\
\hline Target case & 21 & $\begin{array}{c}\text { lubricating oil } \\
\text { leak }\end{array}$ & $\begin{array}{c}\text { HP4, Pompe_ } \\
\text { HP }\end{array}$ \\
\hline
\end{tabular}

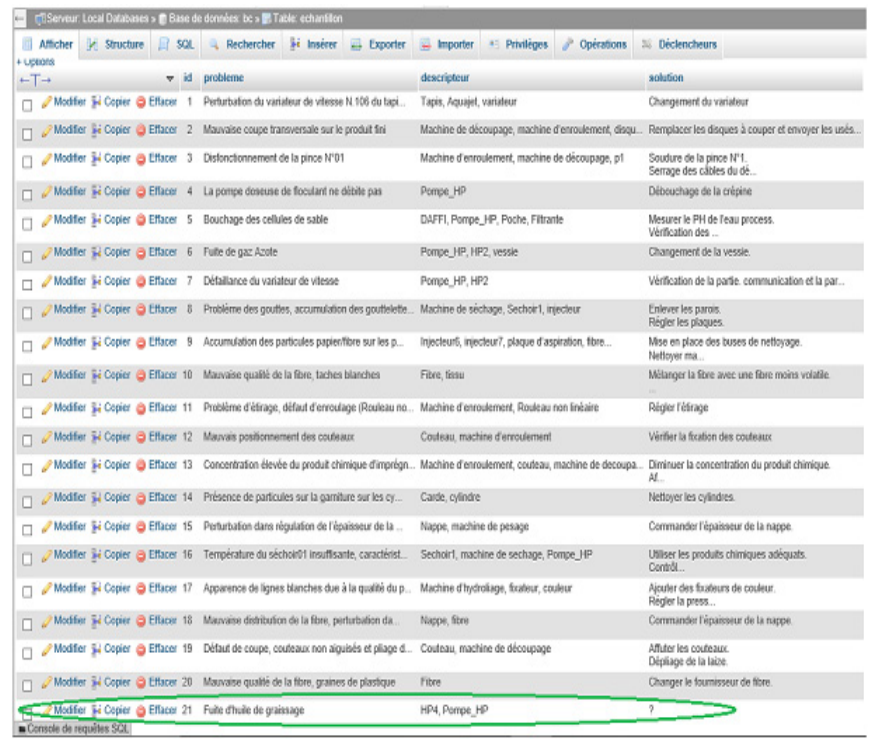

Fig. 7. Sample of source cases.

To compare the relevance of the measures, we rely on the relevance of the following indicators.

Precision: is the fraction of retrieved instances that are relevant; it measures the system's ability to reject irrelevant solutions.

Recall: which calculates the ratio of relevant results existed in relation to relevant results not existed; it measures the system's ability to provide all relevant solutions.

The F-measure: is used for combining and weighing the two indicators accuracy and recall. It measures the system's ability to provide all relevant solutions and reject others.

\section{1) Example according to [17]}

Table 7 shows the results obtained during calculations of similarity between keywords, the similarity threshold is fixed at 0,5 . The similarity is between $[0,1]$, we take values which are greater than (or equal) to 0.5 . Rada measure is presented in equation 2 . It uses a metric dist (c1, c2), which indicates the minimum number of arcs between concept 1 and concept 2 in the domain ontology.

\section{TABLE VII}

Similarity BetweEn Keywords

\begin{tabular}{|c|c|c|c|}
\hline Keyword/Keyword & Pompe_HP & HP2 & Vessie \\
\hline HP4 & 0,5 & 0,33 & $\mathbf{0 , 2 5}$ \\
\hline
\end{tabular}




$$
\operatorname{Sim}_{\text {Rada }}(c 1, c 2)=1 / 1+\operatorname{dist}(c 1, c 2)
$$

dist $($ Vessie;HP4) $=3$

$\operatorname{Sim}_{\text {Rada }}($ Vessie; HP4 $)=1 / 1+\operatorname{dist}($ V essie;HP4 $)=\mathbf{0 , 2 5}$.

The proposed solutions to the problem of lubricating oil leaking are:

1. Draining the strainer,

2. Measuring the $\mathrm{PH}$ of water process, checking the dosing pumps chemicals, checking the ADT system, putting the system in manual mode, making the cons cell washing, changing the filter bags of 05 microns. Table 8 presents evaluation results of Rada measure.

TABLE VIII

Detailed Evaluation of [17] Measure According to Precision (P), Recall (R) AND F-MEAsure (F)

\begin{tabular}{|c|c|c|}
\hline Precision & Recall & F-measure \\
\hline $50 \%$ & $12,5 \%$ & $20 \%$ \\
\hline
\end{tabular}

\section{2) Example according to [18]}

Table 9 shows the results obtained during calculations of similarity between keywords. Wu measure is presented in equation 3, where N1 represents number of arcs between concept 1 and generalizing concept, $\mathrm{N} 2$ is number of arcs between concept 2 and generalizing concept, N3 is number of arcs between generalizing concept and the root in the domain ontology.

TABLE IX

Similarity Between Keywords

\begin{tabular}{|c|c|c|c|}
\hline Keyword/ Keyword & Pompe_HP & HP2 & Vessie \\
\hline HP4 & 0,67 & 0,80 & $\mathbf{0 , 5 4}$ \\
\hline
\end{tabular}

$\operatorname{Sim}_{w \& p}(c 1, c 2)=2 * N 3 / N 1+N 2+(2 * N 3)$

N1 (Vessie, Pompe_HP) $=2$

N2 $($ HP4, Pompe_HP) $=2$

N3 (Pompe_HP) $=4$

$\operatorname{Sim}_{\text {W\&P }}($ Vessie; HP4) $=(2 * 4) /(1+2+2 * 4)=\mathbf{0}, 54$.

The solutions (ordered by number of keywords which contain) to the problem of lubricating oil leaking are:

1. Changing the bladder,

2. Checking the communication and power part,

3. Draining the strainer,

4. Measuring the $\mathrm{PH}$ of process water; checking the dosing pumps chemicals; checking the ADT system; putting the system in manual mode; making the cons cell washing; changing the filter bags of 05 microns.

We get the results shown in Table 10 .

\section{TABLE X}

Detailed Evaluation of [18] Measure According to Precision (P), Recall (R) AND F-MEASURe (F)

\begin{tabular}{|c|c|c|}
\hline Precision & Recall & F-measure \\
\hline $50 \%$ & $25 \%$ & $33,33 \%$ \\
\hline
\end{tabular}

\section{3) Example according to [19]}

The results presented below are not affected by the variation of similarity threshold. The value obtained by [19] as a given concept is relative to domain ontology. Seco measure is presented in equation 4 where hypo(c) represents the number of hyponym concepts, maxwn indicates the number of concepts of the domain ontology (shown in Table 11).

TABLE XI

SIMILARITY BETWEEN KEYWORDS

\begin{tabular}{|c|c|c|c|c|}
\hline Keyword & Pompe_HP & HP2 & Vessie & HP4 \\
\hline Value & 0,331 & 0,294 & $\mathbf{0 , 2 6 2}$ & 0,294 \\
\hline
\end{tabular}

$\operatorname{Sim}_{\text {Seco }}=1-\left(\log (\operatorname{hypo}(c)+1) / \log \left(\max _{w}\right)\right.$

$\operatorname{Max}_{\text {ontology }}=200$

Hypo $($ Vessie $)=6$

$\operatorname{Sim}_{\text {Seco }}=\mathbf{0 , 2 6 2}$

The solutions to lubricating oil leaking are:

1. Changing the bladder.

2. Checking the communication and power part.

3. Draining the strainer.

4. Measuring the $\mathrm{PH}$ of process water; checking the dosing pumps chemicals; checking the ADT system; putting the system in manual mode; making the cons cell washing; changing the filter bags of 05 microns.

Table 12 shows the evaluation results of Seco measure.

TABLE XII

Detailed Evaluation of [19] Measure According to Precision (P), Recall (R) AND F-MEASURE (F)

\begin{tabular}{|c|c|c|}
\hline Precision & Recall & F-measure \\
\hline $50 \%$ & $25 \%$ & $33,33 \%$ \\
\hline
\end{tabular}

In the graph below (Fig.8), the three lines may represent the performance of different measures. When the curve is high, this demonstrates that the concentration of relevant solutions is important. $\mathrm{Wu}$ measure and Seco are winner of this comparison, at least until the recall rate of $25 \%$. Measuring Rada is well below $\mathrm{Wu}$ and Seco. This study was conducted on a set of event sources including 8 relevant and 12 irrelevant solutions.

\section{Comparison between similarity measures according to 0,5 threshold}

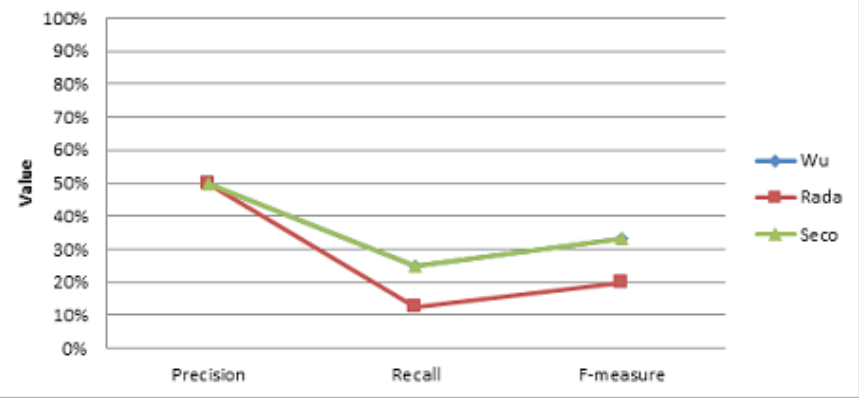

Fig. 8. Comparison results between semantic similarity measures.

Fig.9 shows number of retrieved solutions for each semantic measure. We can see that $\mathrm{Wu}$ and Seco retrieve same number of solutions ( $25 \%$ relevant solutions and about $17 \%$ irrelevant solutions), Rada et al has about 13\% relevant solutions and 9\% irrelevant ones from the cases-base. 
TABLE XIII

Comparison With Other Industrial Systems [1]

\begin{tabular}{|c|c|c|c|c|c|}
\hline Reference & {$[20]$} & [21] & [22] & [1] & Our system \\
\hline Year & 2003 & 2002 & 2009 & $\operatorname{xxxx}$ & 2016 \\
\hline $\begin{array}{l}\text { Distinction between detection } \\
\text { and analysis steps }\end{array}$ & Yes & Yes & No & Yes & No \\
\hline Detection step & Distributed & Distributed & - & Distributed & - \\
\hline Analysis step & Centralized & Distributed & - & Distributed & Centralized \\
\hline $\begin{array}{l}\text { Cooperation between agents for } \\
\text { collective decision making }\end{array}$ & No & No & Yes & Yes & Yes \\
\hline Application domain & Industrial & Industrial & Medical & Industrial and medical & Industrial \\
\hline
\end{tabular}

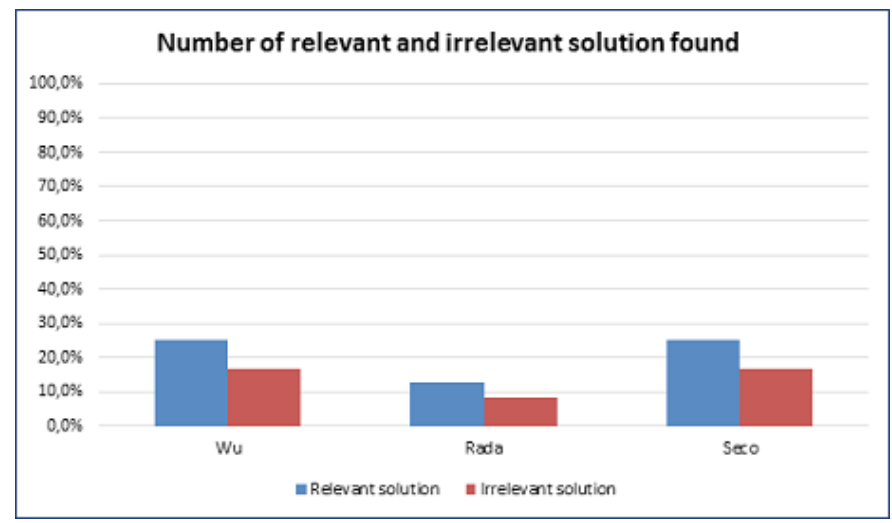

Fig. 9. Number of relevant and irrelevant solution found by each measure.

\section{B. Comparison with other diagnosis systems}

There are several studies that use multi-agent systems in order to solve the diagnosis problem in several domains.

We compare our system with four (4) other systems dedicated to the diagnostic task: MAGIC (Multi-Agent-based Diagnostic Data Acquisition and Management in Complex Systems) [20] DIAMOND (Distributed Architecture for MONitoring and Diagnosis) [21], CMDS (Contract Net based Medical Diagnosis System) [22] and [1] based on the criteria listed below.

Distinction between detection and analysis steps: [20], [21] systems distinguish between detection step and analysis step for that they combine two different types of agents, [22] the system doesn't distinct between these two steps. As the [20] system [1] distinguish between the detection and analysis steps. Our system does not differentiate between these two steps of diagnosis.

The detection step is distributed on dedicated agents called diagnostic agents in MAGIC and control agents in DIAMOND. It is also distributed in the system proposed by Allem where various agents are called detection agents. Our system does not detect defects on the production process.

The analysis step: in MAGIC location is centralized in the diagnostic decision making agent while in DIAMOND is distributed on various diagnostic agents. In [1], it is also distributed on different analysis agents. In our system the task is supported by the operator agents then transmitted to the manager agent.

Cooperation between agents for collective decision making: MAGIC in decision making is performed by a single agent while in DIAMOND. It is distributed over several diagnostic agents. However, there is a real cooperation for the diagnosis overall. CMDS system describes a cooperative problem resolution by medical agents. In [1], analysis agent interacts with other agents of the same type to calculate a cooperative manner in its own local diagnosis. Our problem solving system is shared between the operator agent, manager agent, similarity agent and knowledge agent.

Scope of application: MAGIC and DIAMOND systems are dedicated to the industrial diagnostic, CMDS is specialized into medical diagnosis. In [1], system can be used in the both. Our system is dedicated only to the industrial domain.

After identifying the problem, nonwovens operators can use the developed CDSS [3] in order to find some solutions to their problem in two several ways. First, throughout case based reasoning, it compares the target case to source cases based on similarity measure. Second, it invites operators to collaborate together and solve their problem. Our system requires negotiation strategy when operators have a conflict situation. All agents cooperate for a final decision making.

In comparison with other works that were previously mentioned, the system that we proposed is mainly based on a new collaborative decision-making process. Our objective is to involve operators, experts, and managers who are working in the field of nonwoven industry in the whole process.

Here, collaboration is done in two phases: the first is used to generate solutions to the various problems that are posted by the operators. The second is related to the negotiation of some operators with others or with the experts for the classification of solutions taking into account the operators preferences for the final vote.

In our system, the operator is at the center of reflections on the future of the company. We give him a great deal of participation in decision making. The senior managers of INOTIS enterprise rely on the group's decision and not on personal positions.

The case-based reasoning used in our system is structured on the knowledge represented in ontological form. The domain ontology exploited solutions during the search phase which is dedicated to the field of nonwovens. It is created, tested, and validated by domain experts.

So, our system provides a space for collaboration and knowledge capitalization tool.

\section{System complexity}

Here, we present a theoretical complexity which is calculated based on the messages exchanged in our negotiation protocol. We don't take into consideration the kind of platform and the living messages that are exchanged by the operator agents. However, we rely on the other messages of vote.

Complexity is an important feature for negotiation. We consider the complexity in number of messages induced by our protocol.

In the worst case, the number of messages can be $O(m){ }^{n}$ where 
$\mathrm{n}$ is the depth of the cascade process and $\mathrm{m}$ is the number of agents involved in the negotiation.

Suppose $\mathrm{m}$ agents are involved in the negotiations ( 1 manager agent, 1 agent similarity, 1 Knowledge agent, and $\mathrm{m}-3$ operators). We should take into consideration that there is no equality between the solutions.

The manager provides a list of solutions in which nonwovens operator scores each solution. After that, the manager diffuses the ordered list of solutions: $3 *(\mathrm{~m}-3)+2$ messages are exchanged, including one message to recover the pre-ordered list and another to update $\mathrm{CB}$ by knowledge agent.

Once there is a gender solution, the manager asked for another vote to operators who turn solutions' scores. $2 *(\mathrm{~m}-3)$ messages are exchanged. The manager sends a final list of solutions, which adds $3 *$ (m - 3) messages.

A total of $5 *(m-1)+2$ messages are exchanged, taking account of the first proposal of the manager and the operators responses where at least one is negative. The manager sends $3 *(\mathrm{~m}-3)+2$ messages and receives $2 *(\mathrm{~m}-3)+1$. Each operator receives three (3) messages and sends two (2).

Ordered solutions, with or without equal solutions, has an overall complexity of $\mathrm{O}(\mathrm{m})$, straight to the manager and $\mathrm{O}(1)$ for operators.

\section{Agents'performances}

Although an agent is defined as an autonomous capable entity of acting alone, it is consider as a program that can at any time bug. To address this situation, we save agents states (operator agent, manager agent, similarity agent, and knowledge agent). Each agent is able to recover its saved state.

The agent manager represents the most important agent in our system. It executes many instructions and has a long run time.

\section{Conclusion and Future Works}

The work presented in this paper is a real contribution to the problems resolution of diagnosis in nonwovens industry.

In fact, diagnosis system is the method which determines whether the industrial system is affected by any defect and discriminates the cause of the fault.

Aiming to build a system that provides solutions to industrial problems, we opted for a case-based reasoning (CBR). It is proposed as an alternative to use reasoning rules, costly in terms of learning. More generally, we found a distinction between two motivations for CBR system: first, analogic heuristic, which is chosen for its effectiveness (in terms of computation time, etc.). Secondly, heuristic which is interested in the outcome of reasoning rather than the effectiveness of the reasoning.

During this work, we tried to combine the two approaches to benefit from the speed calculations with multi- agent system on one side and also to the quality of solutions that are based on the similarity measure of [18]. All this work will provide some answers as quickly as possible to the nonwovens operators who are often impatient to finalize their diagnostic problems. Besides, our system aims to classify the provided solutions by its operators through a trading strategy including both operators "preferences (preferences module), and the information content of solutions.

The results presented in this paper provide the basis for future research in several areas. Firstly, when CBR systems are applied to real-world problems as it is for us (nonwovens industry), retrieved solutions can rarely be directly used as adequate solutions for each new problem. Retrieved solutions, in general, require adaptations in order to be applied to new contexts.
Secondly, one future direction of our work is to develop and extend the model of agents allowing them to change their goals. For this reason, we aim to develop an argumentation based strategy of negotiation, which will be more flexible than the voting method but it requires a greater reasoning mechanism that is incorporated in the agents.

Thirdly, it will be very interesting to test our system in other nonwovens company located in Algeria in order to obtain a feedback on the usability of the developed CDSS.

\section{REFERENCES}

[1] K. Allem, R. Maamri, Z Sahnoun. «Un Modèle SMA pour le Diagnostic Collectif.»

[2] INOTIS. (2012, January 9).INOTIS enterprise [Online]. Available: http:// www.inotis.com

[3] F.Z. Benkaddour, N. Taghezout, B. Ascar, "Towards a Novel Approach for Enterprise Knowledge Capitalization Utilizing an Ontology and Collaborative Decision-Making: Application to Inotis Enterprise.'International Journal of Decision Support System Technology (IJDSST) 8.1 (2016): 1-24.

[4] Kolodner, L. Janet, "An introduction to case-based reasoning." Artificial Intelligence Review 6.1 (1992): 3-34.

[5] Pal, K. Sankar, and C.K Simon, Shiu, Foundations of soft case-based reasoning. Vol. 8. John Wiley \& Sons, 2004.

[6] S. H. Ji, M. Park, H. S. Lee, Y. S. Yoon, "Similarity measurement method of case-based reasoning for conceptual cost estimation." Proceedings of International Conference on Computing in Civil and Building Engineering. 2010.

[7] I. Rasovska, Contribution à une méthodologie de capitalisation des connaissances basée sur le raisonnement à partir de cas: Application au diagnostic dans une plateforme d'e-maintenance. Diss. Université de Franche-Comté, 2006.

[8] C. El Aoun, H. B. Ayed, H. Eleuch, E. Aïmeur, F. Kamoun, «Le Raisonnement à Base de Cas Appliqué à la Négociation Electronique.» 5th International Conference: Sciences of Electronic, Technologies of Information and Télécommunications (SETIT 2009). 2009.

[9] J. Cai, Y. Jia, C. Gu, W. Wu,"Research of wartime equipment maintenance intelligent decision-making based on case-based reasoning." Procedia Engineering 15 (2011): 163-167.

[10] P. P. Ruiz, D. Noyes, B. Kamsu-Foguem, «Raisonnement collaboratif à partir de cas dans la résolution de problèmes en maintenance.»9th International Conference on Modeling, Optimization \& Simulation. 2012.

[11] R. H. C. Palácios, I. N. da Silva, A. Goedtel, W. F. Godoy, "A novel multiagent approach to identify faults in line connected three-phase induction motors." Applied Soft Computing45 (2016): 1-10.

[12] M. J. Mendes, J. M. Calado, J. S. da Costa, "Fault diagnosis system based in agents." Fault Detection, Supervision and Safety of Technical Processes. Vol. 6. No. 1. 2006.

[13] J. Pitt, L. Kamara, M. Sergot, A. Artikis, "Voting in multi-agent systems." The Computer Journal 49.2 (2006): 156-170.

[14] M. A. Mahmoud, M. S. Ahmad, M. Z. M. Yuso, A. Idrus, «An Automated Negotiation-based Framework via Multi-Agent System for the Construction Domain." International Journal of Artificial Intelligence and Interactive Multimedia 3.5 (2015): 23-27.

[15] H. B. Baron, M. M. Rojas, R. G. Crespo, O. S. Martinez, "A multiagent matchmaker based on hidden markov model for decentralized grid scheduling." Intelligent Networking and Collaborative Systems (INCOS), 2012 4th International Conference on. IEEE, 2012.

[16] E. Torres, O. Sanjuan, L. Joyanes, J. D. García, R. González, "An Architecture For Management Of Distributed And Redundant Web Storage With Ingelligent Agent Systems And Emerging Techniques." IEEE Latin America Transactions 6.6 (2008): 524-528.

[17] R. Rada, H. Mili, E. Bicknell, M. Blettner, "Development and application of a metric on semantic nets."Systems, Man and Cybernetics, IEEE Transactions on 19.1 (1989): 17-30.

[18] Z. Wu, M. Palmer, "Verbs semantics and lexical selection."Proceedings of the 32nd annual meeting on Association for Computational Linguistics. Association for Computational Linguistics, 1994.

[19] N. Seco, T. Veale, J. Hayes. "An intrinsic information content metric for 
semantic similarity in WordNet." ECAI. Vol. 16. 2004.

[20] B. Köppen-Seliger, T. Marcu, M. Capobianco, S. Gentil, M. Albert, S. Latzel, "MAGIC: An integrated approach for diagnostic data management and operator support." Fault Detection, Supervision and Safety of Technical Processes 2003 (SAFEPROCESS 2003): A Proceedings Volume from the 5th IFAC Symposium, Washington, DC, USA, 9-11 June 2003. Vol. 1. Elsevier, 2004.

[21] M. Albert, T. Längle, H. Woern. Development tool for distributed monitoring and diagnosis systems. KARLSRUHE UNIV (GERMANY FR), 2002.

[22] B. L. Iantovics, "Cooperative Medical Diagnosis Elaboration by Physicians and Artificial Agents." From System Complexity to Emergent Properties. Springer Berlin Heidelberg, 2009. 315-339.

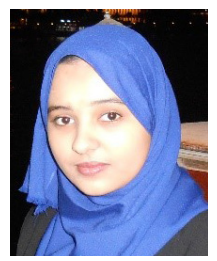

F. Z. Benkaddour received her Master on ID-IHM Degree from university of Oran 1 Ahmed BenBella, Algeria in 2013. She is a member of the EWG-DSS (Euro Working Group on Decision Support Systems) since May 2015. She is currently a Ph.D. student at the same university. Her research topics include collaborative decision support system, knowledge management, case-based reasoning systems, multi agents systems, and WEB technologies. Her last work was published in International Journal of Decision Support System Technology (IJDSST).

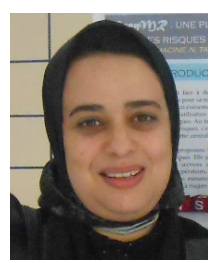

$\mathbf{N}$. Taghezout is an assistant professor at university of Oran 1 Ahmed BenBella, Algeria. She holds her doctorate thesis in MITT at PAUL SABATIER UNIVERSITY in France in 2011. She also received another doctorate thesis in Distributed Artificial Intelligence from university of Oran 1 Ahmed BenBella in 2008. She holds a Master degree in Simulation and Computer aided-design. She conducts her research at the LIO laboratory as a chief of the research group in Modeling of enterprise process by using agents and WEB technologies. Since she studied in UPS Toulouse, she became a member of the EWG-DSS (Euro Working Group on Decision Support Systems). She is currently lecturing Collaborative decision making, Enterprise management and Interface human machine design. Her seminars, publications and regular involvement in Conferences, journals and industry projects highlight her main research interests in Artificial Intelligence.

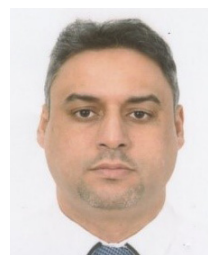

B. Ascar is currently a deputy general manager at INOTIS since April 2009. He obtained his Marine Engineer degree in 2000, from Mechanical Engineering Faculty at Oran University of science and technology USTO. He is a member of EDANA (international association serving the nonwovens and related industries). His is mainly interested in integration of intelligent systems in industrial processes. 